\title{
In vivo biocompatibility of boron nitride nanotubes: Effects on stem cell biology and tissue regeneration in planarians
}

Alessandra Salvetti ${ }^{1 \&}$, Leonardo Rossi ${ }^{1 \&}$, Paola Iacopetti $^{1}, \mathrm{Xia} \mathrm{Li}^{2}, \mathrm{Simone} \mathrm{Nitti}^{3}$, Teresa Pellegrino ${ }^{3}$, Barbara Mazzolai ${ }^{4}$, Virgilio Mattoli ${ }^{4}$, Dmitri Golberg ${ }^{2}$, Gianni Ciofani ${ }^{*}$

1 University of Pisa, Department of Clinical and Experimental Medicine, Via Alessandro Volta 4, 56126 Pisa, Italy

2 National Institute for Materials Science (NIMS), International Center for Materials Nanoarchitectonics (MANA), Namiki 1-1, 305-0044 Tsukuba (Ibaraki), Japan

3 Istituto Italiano di Tecnologia, Via Morego 30, 16163 Genova, Italy

4 Istituto Italiano di Tecnologia, Center for Micro-BioRobotics@SSSA, Viale Rinaldo Piaggio 34, 56025 Pontedera (Pisa), Italy

* gianni.ciofani@iit.it; Tel. +39050883019; Lab. +39050883027; Fax +39050883497

\& AS and LR have equally contributed to this work 


\begin{abstract}
Aims. Boron nitride nanotubes (BNNTs) represent an extremely interesting class of nanomaterials, and recent findings have suggested a number of applications in the biomedical field. Anyhow, extensive biocompatibility investigations are mandatory before any further advancement toward pre-clinical testing. Materials \& Methods. Here, we report on the effects of multi-walled BNNTs in freshwater planarians, one of the best-characterized in vivo models for developmental biology and regeneration research. Results and Discussion. Obtained results indicate that BNNTs are biocompatible in the investigated model, since they do not induce oxidative DNA damage and apoptosis, and do not show adverse effects on planarian stem cell biology and on de-novo tissue regeneration. In summary, collected findings represent another important step toward BNNT realistic applications in nanomedicine.
\end{abstract}

\title{
Keywords
}

Boron Nitride Nanotubes; Planarians; DNA damage; Oxidative stress; Blastema 


\section{Introduction}

Boron nitride nanotubes (BNNTs) represent an innovative and intriguing class of nanomaterials that, thanks to their impressive chemical and physical properties, presume plenty of applications in the nanotechnology field [1, 2]. As recently reviewed [3], the studies related to their biocompatibility and possible exploitation in biomedicine have started to get a full attention. In fact, BNNTs have been proposed as smart nanoparticles both as nanovectors for drug delivery purposes [4], and as intracellular nanotransducers [5].

Although several data obtained in vitro using different cell lines indicate no adverse effects of BNNTs, only a few pilot investigations have been performed in vivo on a limited number of animals (rabbits), that pointed out no toxic effects on blood, liver, and kidney functionality [6, 7]. Furthermore, an ex-vivo biodistribution study in mice showed that BNNTs were mainly accumulated in the liver, spleen, and intestinal tissues, and eliminated via renal extraction [8]. To the best of our knowledge, no further data are available concerning in vivo effects of BNNTs on biological processes.

Freshwater planarians represent a perfect model organism for in vivo studies of stem cells and tissue regeneration [9-12], and they are also largely exploited in pharmacological investigations [13-15], as well as in toxicological studies of organic pollutants [16-19]. Recently, planarians have also been used to analyze the toxicity of silver nanoparticles [20].

Planarians are characterized by a great regeneration capability, as they are able to restore the missing body parts from any small fragment of their body [12]. This intriguing capability is due to the presence of a heterogeneous and abundant population of adult stem cells, called neoblasts, that are spread through the planarian body, with the exception of the most anterior end of the head [21]. Neoblasts are the only proliferating cells in planarians, and among them some clonogenic pluripotent cells (cneoblasts) are able to form descendant-cell colonies in vivo as recently identified 
[22]. After amputation, neoblasts proliferate and accumulate below the wound epithelium, giving rise to an unpigmented structure called blastema that, in the case of head regeneration, is largely devoid of proliferating cells [23]. Remodeling of pre-existing tissues and neoblast differentiation at the wound site give rise to tissue regeneration.

Here we report on the effects of BNNTs in planarians. Our data indicates that BNNTs are biosafe as they do not induce oxidative DNA damage and apoptosis. Moreover, BNNTs have no effects on stem cell biology and on de-novo tissue regeneration.

\section{Methods}

\section{BNNT dispersion preparation}

High-purity (90\%) and almost perfect crystalline multi-walled BNNTs were obtained through a carbon-free chemical vapor deposition technique, by using boron and metal oxides as reactants at about $1,500{ }^{\circ} \mathrm{C}$, as previously described [24]. The final product revealed nanotube lengths up to 10 $\mu \mathrm{m}$, and external diameters in the range $10-80 \mathrm{~nm}[25]$.

Shortening and stabilization of BNNTs in aqueous environment were achieved through a homogeneization / ultrasonication procedure widely described in the literature [26]. Briefly, BNNTs (1 mg) were mixed with $1 \mathrm{ml}$ of a $0.1 \%$ gum Arabic (Sigma) solution; the mixture was homogenized for $15 \mathrm{~min}$ at 30,000 rpm with a homogenizer (T10 basic, UltraTurrax) and sonicated for $24 \mathrm{~h}$ (Bransonic sonicator 2510) using an output power of $20 \mathrm{~W}$. Obtained dispersions were observed and analyzed using scanning electron microscopy (FEI Helios 600) and transmission electron microscopy (Jeol $100 \mathrm{SX}$ ). Size distribution was determined through dynamic light scattering with a Malvern Zetasizer Nano S90.

\section{Animals}


Planarians used in this work belong to the species Dugesia japonica, asexual strain GI [27]. Animals were kept in autoclaved stream water at $18{ }^{\circ} \mathrm{C}$, and starved for at least 2 weeks before being used experiments (starvation is a common practice in planarian laboratories to avoid interference of food with the experimental procedures). Regenerating fragments were obtained by transection between auricles and pharynx, or immediately behind the pharynx.

For BNNT treatment, animals were injected into the gut with gum Arabic coated BNNTs using the Nanoject Microinjectior (Drummond). To determine the short-term effect (acute), animals received a single injection of 100 or $200 \mu \mathrm{g} / \mathrm{g}$ of BNNTs and were processed for experiments 4 or $24 \mathrm{~h}$ after the injection. To determine the long-term effect (chronic), animals were injected twice a week for 15 days to receive a total amount of 100 or $200 \mu \mathrm{g} / \mathrm{g}$ of gum Arabic coated BNNTs. For mitosis analysis and Comet assay experiments, animals received a single injection of 100 or $200 \mu \mathrm{g} / \mathrm{g}$ of BNNTs daily, for three consecutive days, and were then processed for analysis. For elemental analysis, animals received a single injection of $200 \mu \mathrm{g} / \mathrm{g}$ of BNNTs and were processed 24 and $72 \mathrm{~h}$ after the injection. Control animals were injected with equal doses of vehicle (gum Arabic, 1 $\mathrm{mg} / \mathrm{ml})$.

As a "positive" control of nanotoxicity, zinc oxide nanorods (ZnO NRs, 773999 from Sigma) were also administered to animals, while as a "negative" control, cerium oxide nanoparticles (nanoceria, 544841 from Sigma) were injected. Details about these nanomaterials are reported as Supplementary Material.

For comparing BNNTs with ZnO NRs and nanoceria treatment, animals received a single injection of $200 \mu \mathrm{g} / \mathrm{g}$ of BNNTs or ZnO NRs or nanoceria daily, for three consecutive days. Control animals were injected with equal doses of vehicle (gum Arabic, $1 \mathrm{mg} / \mathrm{ml}$ ). During the treatment, animals were inspected for evident morphological alterations, and counted to quantify mortality. Some specimens were processed for propidium iodide/JC1 staining. 


\section{Transmission electron microscopy}

Transmission electron microscopy (TEM) on animals was performed as previously described in [28], with minor modifications. Briefly, planarians were fixed with $3 \%$ glutaraldehyde and $2 \%$ paraformaldehyde in $0.1 \mathrm{M}$ cacodylate buffer, and post-fixed with $2 \%$ osmium tetroxide for $2 \mathrm{~h}$. Ultrathin sections were stained with uranyl acetate and lead citrate before observation.

Elemental analysis by inductively coupled plasma spectrometry (ICP-AES)

For boron content analysis, 250 planarians per sample were used. One and 3 days after the injection, animals were collected and frozen at $-80^{\circ} \mathrm{C}$. They were subsequently freeze-dried, weighted, and then processed for elemental analysis. The digestion process was carried out through a treatment with a $\mathrm{HNO}_{3}: \mathrm{H}_{2} \mathrm{O}_{2}(4: 1$ in volume) solution using $10 \%$ in volume of acidic solution on the total volume of sample. The mineralized samples were finally dissolved in $6 \mathrm{ml}$ of MilliQ grade water (18.3 $\mathrm{M} \Omega)$, and boron concentration was determined through elemental analysis by an inductively coupled plasma atomic emission spectrometer (ICP-AES spectrometer, iCAP 6500, Thermo); for the quantification of boron content, the most sensitive $249.7 \mathrm{~nm}$ boron emission line was used.

\section{Morphometric analysis}

Regenerating head and tail fragments were treated with $2 \%$ hydrochloric acid for 5 min at $4{ }^{\circ} \mathrm{C}$ and then fixed in $100 \%$ ethanol. Fixed specimens were examined under a Zeiss Axioplan microscope, and images were recorded with a Nikon camera. Digital images were quantified using ImageJ software [29]. Blastema area was determined for at least 35 regenerating animals obtained from two independent experiments. We considered as the blastema area the unpigmented region below the wound epithelium; blastema boundary was manually marked by the operator in blind. 
Control and BNNT-treated animals were dissociated into individual cells in phosphate buffered saline as previously described [30]. Three independent samples, each obtained by pooling 3 animals, were analyzed in duplicate for each experimental condition. Briefly, cell pellets were mixed with $0.6 \mathrm{ml}$ of $0.5 \%$ low melting point agarose, spread over a microscope slide previously coated with a thin layer of $1 \%$ routine agarose, and allowed to firm up at $4{ }^{\circ} \mathrm{C}$ for $10 \mathrm{~min}$. After adding a third low melting agarose layer the cells were placed in lysis solution $(2.5 \mathrm{M} \mathrm{NaCl}, 10 \mathrm{mM}$ TrisHCl, 0.1 M EDTA, $1 \%$ Triton-X-100, $10 \%$ DMSO, pH 10) at $4{ }^{\circ} \mathrm{C}$.

To measure apoptosis, a DNA diffusion assay was performed, as described by Singh [31]; in this case, slides were removed from lysis solution after $30 \mathrm{~min}$. Diffused DNA fragments were detected by staining with the fluorescent dye Hoechst 33342 at the final concentration of $5 \mu \mathrm{g} / \mathrm{ml}$. Apoptotic cells show a circular gradient of granular DNA, with a dense central zone and a lighter and hazy outer zone, giving the overall appearance of a halo. About 200 cells per slide were scored under the axioplan epifluorescence microscope, and the number of apoptotic figures was counted.

For DNA damage analysis, the alkaline Comet assay was applied. In this case, slides were incubated in lysis solution for $1 \mathrm{~h}$, treated with alkaly $(300 \mathrm{mM} \mathrm{NaOH}, 1 \mathrm{mM}$ EDTA) for $10 \mathrm{~min}$, then electrophoresed at $300 \mathrm{~mA}, 25 \mathrm{~V}$ for $5 \mathrm{~min}$, in alkalyne solution. After neutralization, nucleoids were stained with Hoechst 33342, and analyzed under a Zeiss Axioplan microscope; images were recorded with a Nikon camera. Digital images were analyzed using the program ImageJ [29] and percentage of DNA migrated in the tail was recorded.

\section{Propidium iodide/JC1 staining}

Planarians (5 specimens per sample) were cut into tiny fragments, then incubated with $200 \mathrm{U} / \mathrm{ml}$ collagenase and $1 \mu \mathrm{g} / \mathrm{ml}$ DNase I for $15 \mathrm{~min}$ at room temperature, during which the suspension was mixed with a pipette every $5 \mathrm{~min}$. After decanting residual tissue fragments, propidium iodide (PI) and $\mathrm{JC} 1$ were added to the supernatants, at the final concentrations of $2 \mu \mathrm{g} / \mathrm{ml}$ and $1 \mu \mathrm{g} / \mathrm{ml}$, 
respectively, followed by a $15 \mathrm{~min}$ incubation in the dark, at room temperature. Cells were collected by centrifuging at $50 \mathrm{~g}$ for $8 \mathrm{~min}$, then resuspended in $100 \mu \mathrm{l}$ of 5/8 Holtfreter's solution. $40 \mu 1$ of cell suspension were dropped on a microscope slide, and immediately examined under a Zeiss Axioplan microscope: dead cells appeared uniformly stained by PI, while in live cells only mitochondria were clearly and brightly stained by JC1.

\section{Real-Time RT-PCR}

RNA was obtained from two planarians per sample, using the NucleoSpin RNA kit (MachereyNagel), following manufacturer's instructions. RNA was quantified using a Nanodrop spectrophotometer, and $500 \mathrm{ng}$ were retro-transcribed into cDNA, using hexanucleotide randomprimers and Maxima Reverse Transcriptase (Thermo scientific), following manufacturer's instructions. Real-time PCR analysis was performed using the GoTaq qPCR Master Mix (Promega). Transcript levels of the following genes were analyzed: DjMcm2, DjPiwi-A,DjMcp and $D j N b 21$. The expression level of $D j E F 2$ was used as internal reference. Specific primers utilized are indicated in Table 1.

Primers were utilized according to the following protocol: initial denaturation of $10 \mathrm{~min}$ at $95{ }^{\circ} \mathrm{C}$, followed by 40 cycles of $15 \mathrm{~s}$ at $95{ }^{\circ} \mathrm{C}$ and $30 \mathrm{~s}$ at $60^{\circ} \mathrm{C}$. Analysis was carried out in the Eco RealTime PCR System (Illumina); two independent samples for each experimental condition were analyzed.

\section{Phototactic assay}

The phototactic test was used to monitor possible behavioral changes following both acute and chronic treatment with BNNTs and was performed as described in [32], with minor modifications. After an acclimation period of $1 \mathrm{~min}$, the speed of each animal to reach a target quadrant and the time spent inside the target quadrant during the $180 \mathrm{~s}$ test was measured. The target quadrant was located in the dark end of the container opposite to the enlightened side. 


\section{Analysis of mitosis}

Analysis of mitosis was performed on both intact control and BNNT treated animals $(200 \mu \mathrm{g} / \mathrm{g})$. Three independent samples, each obtained by pooling 2 animals, were analyzed in duplicate for each experimental condition. Before being sacrificed, animals were incubated in $0.3 \%$ colchicine dissolved in planarian stream water, for $7 \mathrm{~h}$. The use of colchicine increases the number of mitotic figures, thus improving the statistical validity of the recorded data. Each animal pool was then incubated in $0.75 \mathrm{ml}$ of maceration solution (glycerol, acetic acid, and distilled water at 1:1:13 ratio), containing $5 \mu \mathrm{g} / \mathrm{ml}$ of Hoechst 33342, for at least $16 \mathrm{~h}$, to obtain a suspension of single cells. Two aliquots of $10 \mu \mathrm{l}$ each were spotted onto a slide, and allowed to dry at $37^{\circ} \mathrm{C}$ for $1 \mathrm{~h}$. About 2000 cells for each spot were then scored under an Axioplan epifluorescence microscope (Zeiss), and the number of mitotic figures was counted.

\section{Statistical analysis}

In order to detect differences among BNNT and vehicle treated groups, statistical significance $(p<$ 0.05) of data obtained from the phototactic assay, real-time RT-PCR, morphometric analysis, diffusion and Comet assay were evaluated with a Student's $t$-test for unpaired data.

\section{Results}

\section{Uptake of BNNTs and their effects in intact animals}

We evaluated the effects of BNNTs in vivo in a planarian set-up (Figure $1 \mathrm{~A}$ ) with different doses of nanotubes for short- (acute) and long-term (chronic) exposures. Injected BNNTs were stabilized in aqueous solutions through a non-covalent wrapping of gum Arabic. SEM imaging (Figure 1 B) of the samples revealed well-dispersed nanostructures, the length being comprised between 1.0 and 2.5 $\mu \mathrm{m}$ and having an average length of about $1.5 \mu \mathrm{m}$, (Figure $1 \mathrm{C}$, for details see [26]). TEM imaging confirms typical multi-walled BNNT morphology (a representative nanotube is shown in Figure 1 C), with an average inner and outer diameter of 20 and $50 \mathrm{~nm}$, respectively. The hydrodynamic 
diameter $\left(D_{H}\right)$ distribution obtained by dynamic light scattering is reported in Figure 1D, and resulted in a average value of about $340 \mathrm{~nm}$. From this values, the model described by Nair et al. [33] has been used for the estimation of the nanotube length $L$ corresponding to the experimental $D_{H}$, by exploiting following equation:

$$
D_{H}=\frac{L}{\ln \left(\frac{L}{d}\right)+0.32}
$$

where $d$ is the diameter of the nanotube $(d=50 \mathrm{~nm})$. By using this equation we obtained an average BNNT length of $1.2 \mu \mathrm{m}$, coherently with the SEM observations.

During the period of both acute and chronic exposure to BNNTs, independently from the dose of BNNTs injected, we did not observe morphological abnormalities (Figure 2 A). Similarly, we failed to detect abnormalities when we injected nanoceria (Figure $2 \mathrm{~A}$ ); conversely, ZnO NRs injected animals showed dorsal blisters and lesions (Figure 2 A) and most of them (27/30) died within 5 days from the first injection (references about nanotoxicity of these nanoparticles can be found as Supplementary Material). As expected, a significant increase of necrotic cells was detected in $\mathrm{ZnO}$ NR treated animals but not in BNNT- neither in nanoceria- treated animals, where the number of necrotic cells was comparable to that of controls (Figure $2 \mathrm{~B}$ and $\mathrm{C}$ ).

To analyze the effects of BNNTs on the nervous system, we performed a behavioral test. We failed to detect any behavioral changes in BNNT treated animals, which showed a negative phototaxis, when exposed to light, comparable to that observed in control animals (Figure $2 \mathrm{D}$ and E).

With the aim to verify the uptake of BNNTs we performed TEM analysis. We failed to detect any morphological changes in intestinal cells of BNNT treated animals respect to controls and we found BNNTs inside cytoplasmic vesicles of intestinal phagocytes of treated animals, 1 day after the last injection (Figure $3 \mathrm{~A}$ and $\mathrm{B}$ ). The nanotubes were no more detectable in animals 3 days after the 
last injection. Quantification of boron content in animals through ICP provided results in line with these qualitative observations (Figure $3 \mathrm{C}$ ). At 1 day after the injection, the boron concentration in planarians was $0.022 \pm 0.001 \mathrm{ppm}$, which corresponds to $87 \mathrm{ng}$ of boron per animal, and decreased to $0.015 \pm 0.001 \mathrm{ppm}$, which corresponds to $62 \mathrm{ng}$ of boron per animal, on the third day. Boron content in control, non BNNT-injected animals, resulted $50 \mathrm{ng}$ per animal.

As dividing cells are thought to be very sensitive to external stimuli, we decided to analyze whether the exposure to BNNTs may modify the expression levels of different markers for planarian stem cells and stem cell progeny. In particular, we analyzed, by real time RT-PCR, the expression of: DjMcm2, a marker for proliferating neoblasts [21]; DjPiwi-A, the D. japonica homolog of Smedwi1 [34], that is expressed in all neoblasts [35]; DjNB21.11.e, a marker of early neoblast progeny [36] and DjMcp, a marker of late neoblast progeny [36]. No significant differences in the expression levels of these markers were observed, between control and BNNT-treated animals (Figure 4 A).

To investigate the effect of BNNTs on neoblast proliferation, we measured the proportion of Mphase cells in animals exposed to both chronic and acute exposure to BNNTs and controls. Our data showed the presence of a comparable number of mitotic cells in BNNT-treated and control animals (Figure 4 B).

To analyze whether oxidative stress and apoptosis can be induced by BNNT treatment, we measured oxidative DNA damage and the presence of apoptosis by Comet and DNA diffusion assays, respectively, in intact animals after acute and chronic exposure to BNNTs. In the absence of damage, the DNA is compact and round. Where DNA has breaks, the negatively charged fragments migrate to the anode, conferring to the nucleus the morphology of a comet. So, the use of Comet assay provides a picture of the types of DNA lesions (double strand breaks, single strand breaks, alkali labile sites), and of their repair kinetics. Our data indicate that BNNTs did not induce DNA damage (Figure $4 \mathrm{C}$ and D), and the number of apoptotic cells in BNNT-treated animals was comparable to that of controls (Figure $4 \mathrm{E}$ and F). A similar scenario was observed when animals 
were injected with the dose of $200 \mu \mathrm{g} / \mathrm{g}$ of BNNTs for three consecutive days, and no significant differences were observed between control and treated animals (Figure $4 \mathrm{C}$ and D). Similarly, we detected a number of apoptotic cells in BNNT- treated animals comparable to that of controls (Figure $4 \mathrm{E}$ and $\mathrm{F}$ ).

\section{BNNT effects on tissue regeneration}

To understand whether BNNTs influenced the regeneration process, both short- and long-term BNNT-treated animals were amputated below the head, and daily monitored for 10 days. We failed to detect morphological abnormalities: both acute and chronic BNNT-treated animals correctly closed the wound and produced a blastema the size of which was comparable to that of controls (Figure $5 \mathrm{~A}$ and B). Moreover, the appearance of the eyes, an endpoint of regeneration, occurred without delay in BNNT-treated animals, indicating that morphogenetic processes occurred regularly. Indeed, no significant differences were observed in negative phototaxis with respect to controls, 8 days after amputation (Figure $5 \mathrm{C}$ and $\mathrm{D}$ ).

\section{Discussion}

Applications of boron nitride nanotubes in biomedicine are continuously expanding, but their biocompatibility requires a deep and extensive investigation. Several findings obtained in different cell models, indicate that relatively short BNNTs have no toxic effects [3, 8]; however, to date, in vivo data on BNNT biosafety is still deficient.

In this paper, we report on the effects of ultra-pure, gum Arabic coated BNNTs in vivo using planarian as test organism. The presence of the polymer wrapping leads to the consideration of a possible "shielding effect" of the toxicity of the plain nanomaterial. However, because of the chemical inertness of BNNTs and because of a reasonable dissolution of the non-covalent coating after the internalization in biological compartments, we can confidently consider biosafety of BNNTs due not only to the gum Arabic wrapping, but to the intrinsic nature of the nanomaterial. In 
any case, the polymer coating is mandatory for the stabilization of BNNTs in physiological conditions, thus a polymer + nanotube system should be always considered in their biocompatibility evaluation.

BNNTs are internalized by intestinal cells, within 1 day after the treatment. Only two intestinal cell types are present in planarians: absorptive phagocytes that engulf food particles for intracellular digestion, and secretory goblet cells that release digestive enzymes into the lumen [37-39]. TEM analysis reveals that BNNTs are internalized into phagocytes, and nanoparticle clusters with electron-density comparable to that of BNNTs are predominantly detected in membrane vesicles in the cytoplasm, as previously described for the $\mathrm{C} 2 \mathrm{C} 12$ cell line [40]. The vesicles have a random distribution in the cytoplasm, and organelles are not affected by the administration of BNNTs. Although our data provided no information on the nature of the mechanism responsible for the uptake, the presence of cellular materials inside the membrane vesicles suggests that BNNTs might be internalized via endocytosis. Extensive studies on BNNT endocytosis are still missing; however, Ciofani and coworkers demonstrated that the internalization of poly-L-lysine coated BNNTs by $\mathrm{C} 2 \mathrm{C} 12$ is energy-dependent, as already noticed for polyethylenimine-coated BNNTs in other cell lines [41]. No BNNTs are seen to be taken-up by other differentiated cells, as well as by undifferentiated cells (neoblasts).

BNNTs are biosafe in planarians, as we failed to detect any morphological, as well as behavioral defects after the treatments; the observation that BNNT-treated animals escape light as controls, indicates that no gross morphological effects of BNNTs are present at the level of the nervous system.

BNNTs do not induce DNA damage in planarians, as indicated by Comet assay that we used to quantify genomic damage at the single cell level, by detecting DNA strand breaks. The absence of DNA damage after BNNT treatments is in line with the finding that BNNTs are not internalized in the nuclear compartment, allowing potential hazards for major genotoxicity phenomena due to 
BNNTs to be avoided [3]. Moreover, BNNTs are not cytotoxic and no apoptosis is induced in BNNT-treated animals.

Although we failed to detect BNNT into stem cells, we nonetheless decided to study the effect of these nanotubes on stem cell biology, as dividing cells are very sensitive to external stimuli, and recent evidences suggest a niche-like role for intestinal phagocytes in controlling neoblast biology [42]. We failed to detect any differences in the expression levels of molecular markers specific for stem cells and stem cell progenies, as well as in the number of mitotic cells, indicating that BNNTs have no adverse indirect effects on stem cell biology. Since neoblasts are essential for tissue regeneration, we expected no negative effects on the regeneration process in BNNT-treated animals. Indeed, treated animals produced a normal blastema indicating that, following amputation, neoblasts proliferate and accumulate below the wound epithelium. Moreover, the appearance of the eyes at a time comparable to that of controls, demonstrate no effects of BNNTs in morphogenetic process.

\section{Conclusion}

Collectively, our findings demonstrate that gum Arabic coated BNNTs are biocompatible in planarians, since they do not induce oxidative DNA damage and apoptosis, and do not show adverse effects on animal stem cell biology and de-novo tissue regeneration. The present demonstration of BNNT biosafety in the planarian in vivo model encourages further efforts in BNNT nanomedicine research.

\section{Future perspective}

In this paper we have assessed for the first time BNNT effects on planarians, one of the bestcharacterized in vivo models for developmental biology and regeneration research, and currently being rediscovered as a useful animal model for pharmacology, drug toxicology and 
nanotoxicology studies. The findings obtained are therefore, in our opinion, extremely important and represent a further step toward a translational application of BNNTs in biomedicine.

\section{Acknowledgments}

Authors gratefully thank Claudio Ghezzani for TEM technical assistance.

\section{Summary points}

Preparation of short ultra-pure BNNTs and animal treatment

- Short BNNTs have been stabilized in aqueous gum Arabic solutions, and injected in planarians

- BNNTs were found inside vesicles in the cytoplasm of intestinal phagocytes of treated animals 1 day after the last injection

- BNNTs were no more detectable in animals 3 days after the last injection

\section{BNNT general effects on planarians}

- No morphological abnormalities

- No behavioral changes

- BNNT-injected animals show an usual negative phototaxis

- No significant differences in the expression levels of stem cells and stem cell progeny markers

- No significant differences in the number of mitotic cells

\section{DNA damage and cell death}

- BNNTs do not induce DNA damage

- Number of apoptotic and necrotic cells in BNNT-treated animals is comparable to that of controls

\section{Effects of BNNTs on animal regeneration}


- No morphological abnormalities during regeneration

- The appearance of the eyes, an endpoint of regeneration, occurs without delay in BNNTtreated animals

- No significant differences were observed in negative phototaxis with respect to controls 8 days after the amputation 


\section{Figure and Table legends}

Figure 1. A) Schematization of the in vivo set-up used for the evaluation of the effects of different doses of multi-walled BNNTs on planarians, upon short- (acute) and long-term (chronic) exposures. Injected BNNTs were stabilized in aqueous solutions through a non-covalent wrapping of gum Arabic. B) SEM image of the BNNT dispersion. C) TEM image of a typical BNNT investigated in this study. C) Hydrodynamic diameter $\left(D_{H}\right)$ distribution obtained by dynamic light scattering.

Figure 2. Effects of BNNTs in intact animals. A) Bright field images of control and treated planarians 3 days after the first injection. Dorsal view, anterior is towards the left. Scale bar: $500 \mu \mathrm{m}$. B) Percentage of necrotic $\left(\mathrm{PI}^{+}\right)$nuclei in planarians treated with BNNTs, nanoceria or $\mathrm{ZnO}$ NRs for three consecutive days and processed $24 \mathrm{~h}$ after the third injection. Control bar: animals injected with equal doses of vehicle. Data represent the mean \pm standard deviation from 3 independent samples; $* p<0.05$. C) Representative nuclei positive for JC1 or propidium iodide (PI); scale bar: $10 \mu \mathrm{m}$. D) Phototactic test used to assess planarian behavior following acute and chronic BNNT exposure. The graph indicates the speed to reach the dark target quadrant. Data represents the mean \pm standard deviation obtained from 10 independent specimens. E) The graph indicates the time spent in the dark target area by animals exposed to acute BNNT treatment $(200 \mu \mathrm{g} / \mathrm{g})$ after 4 and $24 \mathrm{~h}$, and to chronic BNNT treatment (200 $\mu \mathrm{g} / \mathrm{g}$ ), after 2 weeks. Data represents the mean \pm standard deviation from 10 independent specimens.

Figure 3. In vivo BNNT uptake analysis. A) TEM micrograph of an intestinal cell from a BNNTtreated animal; scale bar: $1 \mu \mathrm{m}$. B) Magnification of the box in A; scale bar: $0.2 \mu \mathrm{m} . \mathrm{C})$ The graph indicates boron amount in BNNT-treated planarians as assessed by ICP-AES at 1 and 3 days since treatment, compared to control animals. 
Figure 4. In vivo effects of acute and chronic BNNT exposure on cell proliferation, DNA damage and cell death. A) Real-time PCR analysis in acute and chronic BNNT-treated animals (200 $\mu \mathrm{g} / \mathrm{g}$ ) and controls. Expression levels are indicated as relative units, assuming the value of control planarians as 1 . Each value is the mean \pm standard deviation of two independent samples analyzed in duplicate. B) Analysis of mitotic cells in control and BNNT treated planarians. The number of cells able to enter the M-phase of the cell cycle in a temporal window of $7 \mathrm{~h}$ was analyzed. Each value represents the mean \pm standard deviation of three independent samples, counted in duplicate. C) Representative images of nuclei showing different levels of DNA migrated in the tail; scale bar: $10 \mu \mathrm{m} . \mathrm{D})$ DNA damage (\% of DNA in the tail) in planarians treated with a single dose of BNNTs $(200 \mu \mathrm{g} / \mathrm{g})$ and processed after $4 \mathrm{~h}$ (1) or $24 \mathrm{~h} \mathrm{(2),} \mathrm{or} \mathrm{with} \mathrm{three} \mathrm{doses} \mathrm{of} \mathrm{BNNTs} \mathrm{in} \mathrm{three} \mathrm{consecutive} \mathrm{days} \mathrm{and} \mathrm{processed} \mathrm{after} 4$ h (3) or $24 \mathrm{~h}$ (4) after the third injection, or chronically exposed to BNNT for 2 weeks (5), or treated with $25 \mu \mathrm{M} \mathrm{KMnO} 4$ for $20 \mathrm{~min}$ at $4^{\circ} \mathrm{C}$ as a positive control (KMnO4). Data represent the mean \pm standard deviation from 3 independent samples; control bars: animals injected with equal doses of vehicle. ${ }^{*} p<0.01$. E) DNA diffusion assay of nuclei obtained from planarians treated with a single dose of BNNTs $(200 \mu \mathrm{g} / \mathrm{g})$ and processed after $4 \mathrm{~h}(1)$ or $24 \mathrm{~h}$ (2), or with three doses of BNNTs in three days and processed after $4 \mathrm{~h} \mathrm{(3)}$ or $24 \mathrm{~h} \mathrm{(4)} \mathrm{after}$ the third injection, or chronically exposed to BNNT for 2 weeks (5); control bars: animals injected with equal doses of vehicle. Data represent the mean \pm standard deviation from 3 independent samples. F) Representative normal and apoptotic nuclei obtained by DNA diffusion assay; scale bar: $10 \mu \mathrm{m}$.

Figure 5. Effects of BNNTs in regenerating animals. A) Representative 3 day head and tail fragments regenerating a tail and a head, respectively. The blastema is highlighted in yellow. Arrows indicate eye spots; scale bar: $400 \mu \mathrm{m}$. B) Blastema area in regenerating planarians following BNNT treatment 3 days after amputation. Values represent the mean \pm standard deviation from 35 animals. C) Phototactic test used to assess the behavior of planarians 
following acute BNNT exposure. The graph indicates the speed to reach the dark target quadrant by tail and head fragments regenerating a head and a tail, respectively, 8 days after amputation. Data represents the mean \pm standard deviation from 15 independent specimens. D) The graph indicates the time spent in the dark target quadrant by tail and head fragments regenerating a head and a tail, respectively, 8 days after amputation. Data represent the mean \pm standard deviation from 15 independent specimens.

Table 1. Primer sequences for real-time RT-PCR analysis. 


\section{References}

[1] Wang J, Lee CH, Yap YK. Recent advancements in boron nitride nanotubes. Nanoscale 2(10), 2028-2034 (2010)

[2] Kalay S, Yilmaz Z, Sen O, Emanet M, Kazanc E, Culha M. Synthesis of boron nitride nanotubes and their applications. Beilstein J. Nanotechnol. 6, 84-102 (2015)

[3] Ciofani G, Danti S, Genchi GG, Mazzolai B, Mattoli V. Boron nitride nanotubes: biocompatibility and potential spill-over in nanomedicine. Small 9(9-10), 1672-1685 (2013)

[4] Li X, Zhi C, Hanagata N, Yamaguchi M, Bando Y, Golberg D. Boron nitride nanotubes functionalized with mesoporous silica for intracellular delivery of chemotherapy drugs. Chem. Commun. 49(66), 7337-7339 (2013)

[5] Ciofani G, Danti S, D'Alessandro D, et al. Enhancement of neurite outgrowth in neuronal-like cells following boron nitride nanotube-mediated stimulation. ACS Nano 4(10), 6267-6277 (2010)

[6] Ciofani G, Danti S, Nitti S, Mazzolai B, Mattoli V, Giorgi M. Biocompatibility of boron nitride nanotubes: an up-date of in vivo toxicological investigation. Int. J. Pharmaceut. 444(12), 85-88 (2013)

[7] Ciofani G, Danti S, Genchi GG, et al. Pilot in vivo toxicological investigation of boron nitride nanotubes. Int. J. Nanomed. 7, 19-24 (2012)

[8] Soares DCF, Ferreira TH, Ferreira CDA, Cardoso VN, De Sousa EMB. Boron nitride nanotubes radiolabeled with ${ }^{99 m}$ Tc: Preparation, physicochemical characterization, biodistribution study, and scintigraphic imaging in Swiss mice. Int. J. Pharmaceut. 423(2), 489-495 (2012) 
[9] Gentile L, Cebrià F, Bartscherer K. The planarian flatworm: an in vivo model for stem cell biology and nervous system regeneration. Dis. Model Mech. 4(1), 12-19 (2011)

[10] Reddien P. Specialized progenitors and regeneration. Development 140(5), 951-957 (2013)

[11] Rink JC. Stem cell systems and regeneration in planaria. Dev. Genes Evol. 223(1-2), 67-84 (2013)

[12] Rossi L, Salvetti A, Marincola FM, et al. Deciphering the molecular machinery of stem cells: a look at the neoblast gene expression profile. Genome Biol. 8(4), R62 (2007)

[13] Pagán OR, Baker D, Deats S, et al. Planarians in pharmacology: parthenolide is a specific behavioral antagonist of cocaine in the planarian Girardia tigrina. Int. J. Dev. Biol. 56(1-3), 193-196 (2012)

[14] Rawls SM, Patil T, Tallarida CS, et al. Nicotine behavioral pharmacology: clues from planarians. Drug Alcohol Depend. 118(2-3), 274-279 (2011)

[15] Zhang C, Tallarida CS, Raffa RB, Rawls SM. Sucrose produces withdrawal and dopaminesensitive reinforcing effects in planarians. Physiol. Behav. 112, 8-13 (2013)

[16] Knakievicz T, Silveira PA, Ferreira HB. Planarian neoblast micronucleus assay for evaluating genotoxicity. Chemosphere 72(9), 1267-1273 (2008)

[17] Yuana Z, Zhanga J, Menga W, Zhoua Y. Effects of perfluorooctane sulfonate on behavioural activity, regeneration and antioxidant enzymes in planarian Dugesia japonica. Chemi. Ecol. 30(2), 187-195 (2014)

[18] $\mathrm{Li} \mathrm{MH}$. Acute toxicity of benzophenone-type UV filters and paraben preservatives to freshwater planarian, Dugesia japonica. Env. Toxicol. Chem. 94(3), 566-573 (2012)

[19] Zhang X, Zhang B, Yi H, Zhao B. Mortality and antioxidant responses in the planarian (Dugesia japonica) after exposure to copper. Toxicol. Ind. Health 30(2), 123-131 (2014) 
[20] Kustov L, Tiras K, Al-Abed S, Golovina N, Ananyan M. Estimation of the toxicity of silver nanoparticles by using planarian flatworms. Altern. Lab. Anim. 42(1), 51-58 (2014)

[21] Salvetti A, Rossi L, Deri P, Batistoni R. An MCM2-related gene is expressed in proliferating cells of intact and regenerating planarians. Dev. Dyn. 218(4), 603-614 (2000)

[22] Wagner D, Wang I, Reddien PW. Clonogenic neoblasts are pluripotent adult stem cells that underlie planarian regeneration. Science 332(6031), 811-816 (2011)

[23] Wenemoser D, Reddien PW. Planarian regeneration involves distinct stem cell responses to wounds and tissue absence. Dev. Biol. 344(2), 979-991 (2010)

[24] Tang CC, Bando Y, Sato T, Kurashima K. A novel precursor for synthesis of pure boron nitride nanotubes. Chem. Comm. 12, 1290-1291 (2002)

[25] Zhi CY, Bando Y, Tang CC, Golberg D. Effective precursor for high yield synthesis of pure BN nanotubes. Solid State Commun. 135(1-2), 67-70 (2005)

[26] Ciofani G, Del Turco S, Rocca A, et al. Cytocompatibility evaluation of gum Arabic-coated ultra-pure boron nitride nanotubes on human cells. Nanomedicine UK 9(6), 773-788 (2014)

[27] Orii H, Agata K, Watanabe K. POU-domain genes in planarian Dugesia japonica: the structure and expression. Biochem. Biophys Res. Commun. 192(3), 1395-1402 (1993)

[28] Salvetti A, Rossi L, Lena A, et al. DjPum, a homologue of Drosophila Pumilio, is essential to planarian stem cell maintenance. Development 132(8), 1863-1874 (2005)

[29] Abramoff MD, Magelhaes PJ, Ram SJ. Image processing with ImageJ. Biophotonics International 11(7), 36-42 (2004) 
[30] García-Medina S, García-Medina L, Galar-Martinez M, et al. Genotoxicity and oxidative stress induced by cadmium and zinc in the planarian, Dugesia dorotocephala. African Journal of Biotech. 12(25), 4028-4038 (2013)

[31] Singh NP. Apoptosis assessment by the DNA diffusion assay. Methods Mol. Med. 111, 55-67 (2005)

[32] Inoue $\mathrm{T}$, Kumamoto $\mathrm{H}$, Okamoto $\mathrm{K}$, et al. Morphological and functional recovery of the planarian photosensing system during head regeneration. Zoolog. Sci. 21(3), 275-283 (2004)

[33] Nair N, Kim WJ, Braatz RD, Strano MS. Dynamics of surfactant-suspended single-walled carbon nanotubes in a centrifugal field. Langmuir 24(5), 1790-1795 (2008).[34] Reddien PW, Oviedo NJ. Jennings JR, Jenkin JC, Sánchez Alvarado A. SMEDWI-2 is a PIWI-like protein that regulates planarian stem cells. Science 310(5752), 1327-1330 (2005)

[35] Yoshida-Kashikawa M, Shibata N, Takechi K, Agata K. DjCBC-1, a conserved DEAD box RNA helicase of the RCK/p54/Me31B family, is a component of RNA-protein complexes in planarian stem cells and neurons. Dev. Dyn. 236(12), 3436-3450 (2007)

[36] Eisenhoffer GT, Kang H, Sánchez Alvarado A. Molecular analysis of stem cells and their descendants during cell turnover and regeneration in the planarian Schmidtea mediterranea. Cell Stem Cell 3(3), 327-339 (2008)

[37] Forsthoefel DJ, Park AE, Newmark PA. Stem cell-based growth, regeneration, and remodeling of the planarian intestine. Dev. Biol. 356(2), 445-459 (2011)

[38] Garcia-Corrales P, Gamo J. The ultrastructure of the gastrodermal gland cells in the freshwater planarian Dugesia gonocephala s.1. Acta Zool. 67(1), 43-51 (1986)

[39] Ishii S. Electron microscopic observations on the Planarian tissues II. The intestine. Fukushima J. Med. Sci. 12(1), 67-87 (1965) 
[40] Ciofani G, Ricotti L, Danti S, et al. Investigation of interactions between poly-L-lysine-coated boron nitride nanotubes and $\mathrm{C} 2 \mathrm{C} 12$ cells: up-take, cytocompatibility, and differentiation. Int. J. Nanomedicine 5(1), 285-298 (2010)

[41] Ciofani G, Raffa V, Menciassi A, Cuschieri A. Cytocompatibility, interactions and uptake of polyethyleneimine-coated boron nitride nanotubes by living cells: confirmation of their potential for biomedical applications. Biotechnol. Bioeng. 101(4), 850-858 (2008)

[42] Forsthoefel DJ, James NP, Escobar DJ, et al. An RNAi screen reveals intestinal regulators of branching morphogenesis, differentiation, and stem cell proliferation in planarians. Dev. Cell 23(4), 691-704 (2012)

\section{Reference annotations}

[3]** Comprehensive review on biomedical applications of BNNTs.

$[8]^{* *}$ In vivo investigation of BNNTs.

$[11]^{* *}$ Comprehensive review on planarian regeneration.

$[16]^{*}$ Genotoxicity assays in planarians.

$[20]^{* *}$ Planarians as model for nanotoxicological assessment.

[26]* Cytocompatibility evaluation of BNNTs on human cells. 
Figure 1 
Figure 2 
Figure 3 
Figure 4

\section{Figure 5}


Table 1

\begin{tabular}{|l|l|}
\hline \multicolumn{1}{|c|}{ Gene } & \multicolumn{1}{c|}{ Sequences } \\
\hline DjMcm2 & $\begin{array}{l}\text { 5'-GGCAGGTGAAACATTGGGATCA-3' } \\
\text { 5'-GGCTACCGACATTCCTTTGGT-3' }\end{array}$ \\
\hline DjEF2 & $\begin{array}{l}\text { 5'-GCAATCGAAGACGTTCCATGTG-3' } \\
\text { 5'-CCAGGAAAAGTTGTTATAGTCCCAGTTT-3' }\end{array}$ \\
\hline DjPiwi & $\begin{array}{l}\text { 5'-CGTCTGTGTTTTCTATAAGTTCC-3' } \\
\text { 5'-ACTTTTGCTGGAATGTTGTTATTG-3' }\end{array}$ \\
\hline DjMcp & $\begin{array}{l}\text { 5'-TAATACCAGGGACACCAGTAGAAG-3' } \\
\text { 5'-TATAAAAGCTGGGACATCACGAAA-3' }\end{array}$ \\
\hline DjNB.21.11.e & $\begin{array}{l}\text { 5'-CTGGTAAAGAAAGTGAATCTGAAGGT-3' } \\
\text { 5'-ATCTTCCTCGTCTAACTCTGCAAC-3' }\end{array}$ \\
\hline
\end{tabular}

